\title{
ESTUDO DO ESCOAMENTO DO SISTEMA ÓLEO / ÁGUA EM TUBULAÇÕES INDUSTRIAIS
}

\author{
J. H. B.PESSOA ${ }^{1}$, O.CHIAVONE FILHO ${ }^{2}$,A. C. P. PEREIRA ${ }^{2}$, G. L. C. NASCIMENTO ${ }^{2}$, R. M. P. \\ de ANDRADE ${ }^{2}$, V. A. da SILVA Jr \\ ${ }^{1}$ Petrobras - UO-RNCE, Av. Eusébio Rocha, 1000, Natal, RN- Brasil, 59064-100 \\ ${ }^{2}$ Universidade Federal do Rio Grande do Norte, Campus Universitário, Depto. de Engenharia \\ Química, Lagoa Nova, Natal - RN - Brasil, 59066-800 \\ E-mail para contato: jhbpessoa@gmail.com.br
}

\begin{abstract}
RESUMO - Devido a produção crescente de água nos campos de produção de petróleo na região do Rio Grande do Norte e do complexo sistema de escoamento da malha de líquidos até o processo de tratamento, mostrou-se necessário o mapeamento do escoamento do fluido produzido (óleo + água) em termos da sua viscosidade aparente. Isto se deve ao fato da demanda de uma melhor descrição do comportamento reológico. Sendo assim foram coletados dados reais do escoamento em diversas faixas de BS\&W (basicsedimentsandwater) e foram utilizadas metodologias para seleção dos dados. De posse dos dados selecionados foram realizados cálculos da perda de carga por fricção, usando a lei de Darcy-Weisbach, bem como de ajuste de parâmetro, a viscosidade aparente para cada faixa de BS\&W. Portanto, foi possível a descrição do escoamento nas condições de interesse atravésdo gráfico da viscosidade aparente x BS\&W da emulsão para o óleo estudado.
\end{abstract}

\section{INTRODUÇÃO}

A produção de petróleo associada a um alto volume de água produzida apresenta a geração de emulsões, que podem ser de água em óleo (W/O), óleo em água $(\mathrm{O} / \mathrm{W})$ ou, até mesmo, emulsões múltiplas (W/O/W ou O/W/O). Essas emulsões podem ser formadas tanto nos reservatórios como no fundo dos poços e também durante o escoamento, devido ao turbilhonamento do fluido na passagem por válvulas, tubulações e acidentes. Essas emulsões podem gerar inconvenientes como, por exemplo, o aumento da perda de carga em dutos(BASTIDAS, 2007).

Na indústria do petróleo, boas estimativas de viscosidade, perda de carga, gradiente de pressão, tamanho de gotículas e distribuição do tamanho das gotículas de emulsões água em óleo são muito importantes para a modelagem do processo em termos de energia requerida e problemas de garantia de escoamento. Contudo, apesar da importância dessas emulsões na indústria, poucos estudos foram publicados na literatura (KELEŞOĞLU; PETTERSEN; SJÖBLOM, 2012).

Sendo assim torna-se de interesse tanto científico como para o desenvolvimento industrial, o conhecimento mais aprofundado das implicações da formação de emulsões entre a água produzida e o 


\section{9 a 22 de outubro de 2014 \\ Florianópolis/SC}

petróleo para o escoamento de líquidos.

Este trabalho trata da aquisição de dados de escoamento reais de campos de produção, do tratamento matemático dos mesmos bem como o cálculo da viscosidade aparente a partir dos dados tratados e obtenção do gráfico que correlaciona a viscosidade aparente da emulsão ao BS\&W. Tudo isso com objetivo de mapear a viscosidade do petróleo em estudo para utilização em futuros projetos de sistemas de transferência de óleo.

\section{METODOLOGIA DO TRABALHO}

\subsection{Aquisição dos dados de campo}

A aquisição dos dados de campo foi feita a partir de um sistema de aquisição de dados dos instrumentos de processo em tempo real, é possível obter um histórico de cada instrumento em intervalos de tempo determinados pelo usuário.

Para o presente trabalho foi levantado um histórico de um pouco mais de 1 ano (01/02/2013 a 26/01/2014) e foram obtidos os dados de vazão volumétrica de água $\left(\mathrm{m}^{3} / \mathrm{h}\right)$, vazão volumétrica de óleo $\left(\mathrm{m}^{3} / \mathrm{h}\right)$, pressão manométrica na estação de bombeio $\left(\mathrm{kgf} / \mathrm{cm}^{2} \_\mathrm{g}\right)$ e pressão manométrica na estação de recebimento $\left(\mathrm{kgf} / \mathrm{cm}^{2} \_\mathrm{g}\right)$, com um intervalo de tempo de 6 minutos entre um dado e outro.

\subsection{Tratamento dos dados de campo}

Como se tratam de dados reais de transferência de óleo de uma instalação industrial, estes sofrem influências de diversas intermitências do processo, tais como: necessidade de parada de bombeio nas estações, aumento momentâneo na transferência de óleo em detrimento a da água ou vice versa, intervenção do pessoal de operação em válvulas a jusante do sistema de bombeio, entre outras.

Dessa forma, tornou-se necessário um tratamento estatístico dos dados de escoamento, com a finalidade de minimizar essas intermitências e trabalhar com um modelo de escoamento no regime permanente. Este tratamento de dados objetivou a obtenção de um conjunto de dados de vazão cujo desvio padrão não ultrapassasse $5 \%$ da média, expurgando os valores considerados fora de uma faixa de controle.

O sistema onde esse fluido escoa é composto de um oleoduto com diâmetro interno de 15,5 polegadas e com um comprimento de $55006 \mathrm{~m}$, o que corresponde a um volume hidráulico de 6696,2 $\mathrm{m}^{3}$. Sendo assim, os dados de vazão de determinado período com um mesmo perfil de BS\&W do fluido puderam ser classificados em termos de alcançar ou não o tempo de trânsito, que seria o tempo que um determinado volume de controle de fluido percorreria a tubulação, desde a estação de origem até a estação de destino. Essa classificação foi usada na escolha dos dados que foram utilizados no trabalho.

\subsection{Cálculo da viscosidade aparente}


É denominada viscosidade aparente do fluido aquela viscosidade que corresponde a uma determinada perda de carga dentro de uma tubulação, relativa a uma vazão de escoamento. No caso de escoamento de emulsões essa definição é importante pois, na maioria das vezes, é difícil obter em laboratório, a viscosidade que causa o efeito de perda de pressão no escoamento real.

O escoamento de emulsões pode ser tratado tanto como um escoamento bifásico líquido-líquido com escorregamento entre as fases ou sem escorregamento entre as fases. A hipótese sem escorregamento entre as fases é uma das considerações básicas do modelo de escoamento bifásico homogêneo (HAPANOWICZ, 2008).

A viscosidade aparente da emulsão pode exceder de forma substancial tanto a viscosidade da fase oleosa como a viscosidade da fase aquosa. A viscosidade aparente dessas misturas depende de diversos fatores: viscosidade da água e do óleo, fração volumétrica da água, temperatura, distribuição do tamanho das gotículas, quantidade de sólidos no petróleo e taxa de cisalhamento (PLASENCIA; PETTERSEN; NYDAL, 2013).

A perda de carga em um sistema onde se escoa uma emulsão de petróleo e água produzida depende fortemente da fase da emulsão. Em emulsões W/O (água em óleo) a viscosidade do fluido aumenta com o BS\&W causando um aumento na perda de carga por fricção, esse aumento chega até um determinado $\mathrm{BS} \& \mathrm{~W}$ quando a viscosidade cai vertiginosamente para algo em torno da viscosidade da água. Esse ponto onde há a queda da viscosidade é chamado de ponto de inversão e caracteriza a mudança da emulsão W/O para uma emulsão O/W (óleo em água).

Neste trabalho foi utilizada a hipótese sem escorregamento entre fases, utilizando a emulsão como um fluido único e sua viscosidade aparente sendo diferente da viscosidade dos fluidos que a formam, além disso foi estudada apenas a variação da viscosidade aparente com o BS\&W, que exprime a fração volumétrica de água.

Para o cálculo da viscosidade aparente foi utilizada a equação de Darcy-Weisbach (Equação 1) que calcula a perda de carga por fricção numa tubulação em função das características do fluido (massa específica e viscosidade), dados de processo (vazão e temperatura) e características do oleoduto (diâmetro interno, comprimento e rugosidade relativa).

$$
\Delta p=f \frac{L}{D} \frac{\rho v^{2}}{2}
$$

A viscosidade aparece no cálculo do número de Reynolds (Equação 2) que é usado para o cálculo do fator de atrito, dessa forma o método de cálculo da viscosidade relativa é iterativo pois, assume-se um valor inicial para esta viscosidade, calcula-se a perda de carga por fricção e compara-se a perda de carga obtida por cálculo a experimentada com os dados reais do escoamento.

$$
R e=\frac{\rho v D}{\mu}
$$

O fator de atrito foi calculado, no caso do escoamento laminar $(\operatorname{Re}<2100)$, através da Equação 
3. Quando se tratava de escoamento turbulento, foi usada a equação implícita de Colebrook-White (Equação 4) que foi obtida a partir do diagrama de Moody.

$$
\begin{aligned}
& f=\frac{64}{R e} \\
& \frac{1}{\sqrt{f}}=-2 \log _{10}\left(\frac{e}{3,7}+\frac{2,51}{\operatorname{Re} \sqrt{f}}\right)
\end{aligned}
$$

\subsection{Tratamento dos dados de viscosidade aparente}

Novamente com a finalidade de reduzir as oscilações causadas pela operação de transferência dos fluidos, foi decidido fazer um tratamento estatístico das viscosidades aparentes obtidas para cada faixa de BS\&W de trabalho.

Dessa forma, a cada faixa de BS\&W, foi obtido um conjunto de dados de viscosidade aparente, esses dados foram tratados com o objetivo de deixar o desvio padrão do conjunto de dados menor que $10 \%$ da média, expurgando os valores considerados fora de uma faixa de controle.

Também foi tomado o cuidado de identificar as viscosidades calculadas a partir de períodos de operação que atingiram o tempo de trânsito das calculadas a partir de períodos que não atingiram o tempo de trânsito.

\section{RESULTADOS E DISCUSSÕES}

Usando apenas os dados obtidos a partir dos períodos de operação que atingiram o tempo de trânsito e separando os valores de viscosidade aparente por faixa de BS\&W obtivemos as viscosidades aparentes relacionadas no gráfico da Figura 1.

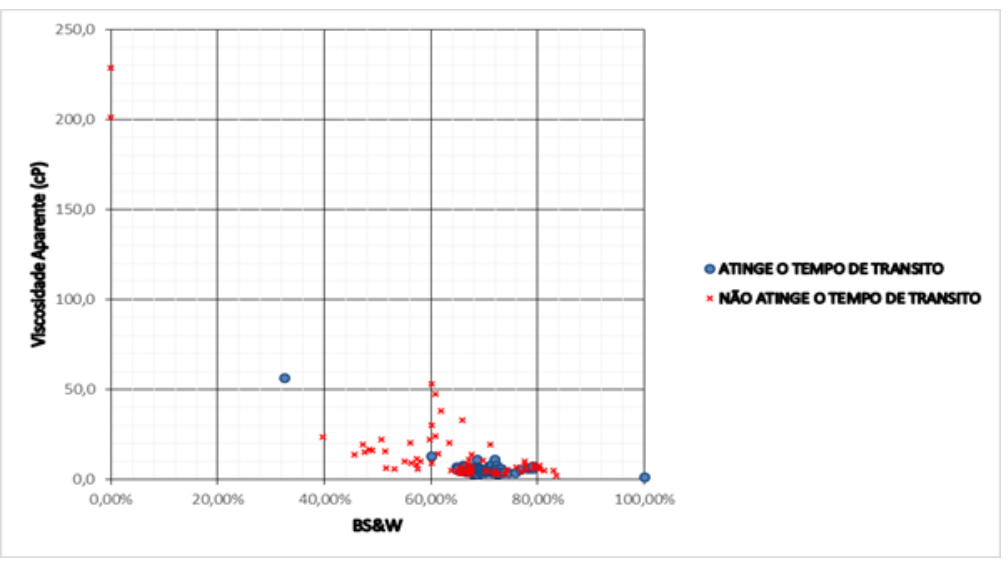

Figura 1 - Viscosidade aparente em função do BS\&W (dados de campo). 
Como se pode observar, o conjunto de dados cujo período de operação atinge o tempo de trânsito segue um comportamento esperado, com a queda da viscosidade aparente em função do aumento do BS\&W.

No entanto, há lacunas onde os dados de operação são pobres ou que não há dados cujos períodos atinjam o tempo de trânsito, sendo assim, para a região onde não temos dados que atinjam o tempo de trânsito mas que há dados, estes foram usados, além disso, como há concentração de dados de viscosidade para determinadas faixas de BS\&W, esses dados foram tratados de modo a reduzir o desvio da média e essa média foi usada para representar a faixa. Esse gráfico é representado na Figura 2.

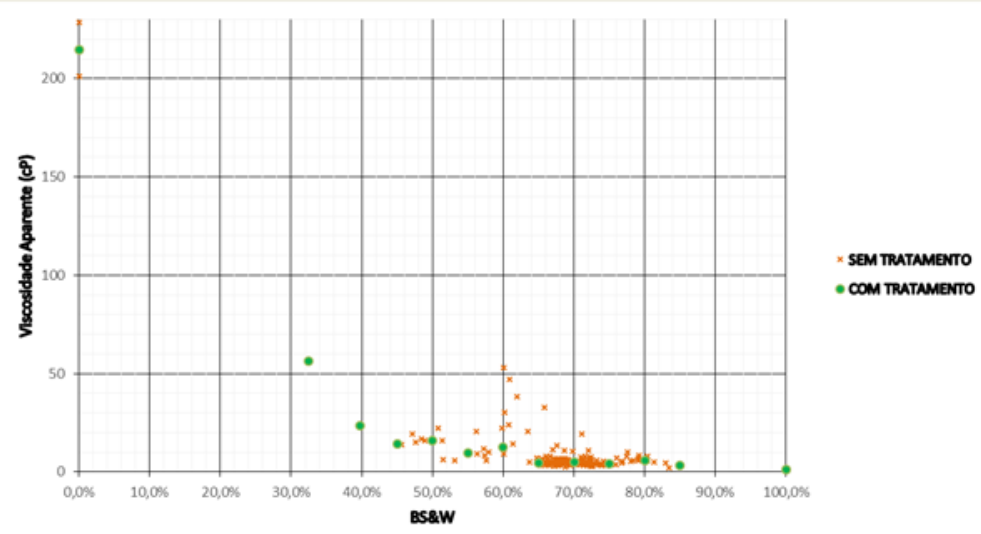

Figura 2 - Viscosidade aparente em função do BS\&W (com tratamento vs sem tratamento).

Depois de realizado o tratamento dos dados nota-se que, mesmo incluindo alguns dados de períodos onde não foi atingido o tempo de trânsito, há uma certa tendência de redução brusca da viscosidade para valores próximos a viscosidade da água $\left(1 \mathrm{cP} @ 20{ }^{\circ} \mathrm{C}\right)$.

Infelizmente, com o conjunto de dados de escoamento do campo também é possível notar que uma região de grande interesse não foi contemplada, a região da inversão de fase que, provavelmente se encontra num BS\&W entre $1 \%$ a $30 \%$. Sendo assim, foram pesquisadas algumas correlações para a obtenção do ponto de inversão de fase e outras para a obtenção da viscosidade aparente, estas correlações são apresentadas na Tabela 1.

Tabela 1 - Correlações usadas para o cálculo do ponto de inversão e da viscosidade aparente

\section{CÁLCULO DO PONTO DE INVERSÃO CÁLCULO DA VISCOSIDADE APARENTE}

\begin{tabular}{|c|c|c|c|}
\hline CORRELAÇÃO & REFERÊNCIA & CORRELAÇÃO & REFERÊNCIA \\
\hline$\varepsilon_{w}^{I}=\frac{1}{1+\left(\frac{\mu_{o}}{\mu_{w}}\right)^{0,5}}(5)$ & (YEH ET AL., 1964) & $\mu_{m}=\varepsilon_{o} \mu_{o}+\varepsilon_{w} \mu_{w}$ & (DUCKLER ET AL., 1964) \\
\hline
\end{tabular}

\begin{tabular}{c|c}
$\boldsymbol{\varepsilon}_{\boldsymbol{w}}^{\boldsymbol{I}}=\mathbf{0 , 5}-\mathbf{0}, \mathbf{1 1 0 8} \log _{\mathbf{1 0}}\left(\frac{\boldsymbol{\mu}_{\boldsymbol{o}}}{\mu_{w}}\right)(\mathbf{6}) \quad \begin{array}{c}\text { (ARIRACHAKARAN } \\
\text { ET AL., 1989) }\end{array}$ & $\mu_{m}=\mu_{o}\left(1-\varepsilon_{w}\right)^{-2,5}(11)$ \\
&
\end{tabular}

(BRINKMAN, 1952) 
$\varepsilon_{w}^{I}=0,3788-0,1108 \log _{10}\left(\frac{\mu_{o}}{\mu_{w}}\right)-$

$9,6533\left(\frac{\rho_{w}-\rho_{o}}{\rho_{w}}\right)^{2}+2,4841\left(\frac{\rho_{w}-\rho_{o}}{\rho_{w}}\right)$

$\varepsilon_{w}^{I}=\left[1+\left(\frac{\mu_{o}}{\mu_{w}}\right)^{1 / 6}\left(\frac{\rho_{o}}{\rho_{w}}\right)^{5 / 6}\right]^{-1}(8)$

$\varepsilon_{w}^{I}=\frac{\left(\frac{\rho_{o}}{\rho_{w}}\right)\left(\frac{\mu_{o}}{\mu_{w}}\right)^{0,4}}{1+\left(\frac{\rho_{o}}{\rho_{w}}\right)\left(\frac{\mu_{o}}{\mu_{w}}\right)^{0,4}}(9)$
(CHEN, 2001)

(7)

(DECARRE; FABRE, 1997)

(BRAUNNER;ULLMA

$\mathrm{NN}, 2002)$

Foram então calculados os pontos de inversão de fase com cada correlação listada e o ponto de inversão de fase usado foi a média obtida entre os pontos de inversão de fase das correlações de Arirachakaran, Decarre e Fabre e Braunner e Ullmann, conforme consta na Tabela 2. As correlações de Yeh e de Chen foram desconsideradas pois são indicadas para escoamento laminar em regime estratificado, o que não se observa no sistema estudado.

Tabela 2 - Cálculo do ponto de inversão de fase

\begin{tabular}{|c|c|}
\hline CORRELAÇÃO & PONTO DE INVERSÃO (\%) \\
\hline YEH ET AL., 1964 & 6,65 \\
\hline ARIRACHAKARAN ET AL., 1989 & 24,58 \\
\hline CHEN, 2001 & 28,00 \\
\hline DECARRE; FABRE, 1997 & 32,19 \\
\hline BRAUNNER; ULLMANN, 2002 & 12,44 \\
\hline MÉDIA & 23,07 \\
\hline
\end{tabular}

Tomando por base o ponto de inversão de fase calculado, foram usados os modelos para cálculo da viscosidade aparente em função do BS\&W da emulsão listados na Tabela 1. Os resultados são apresentados no gráfico da Figura 3.

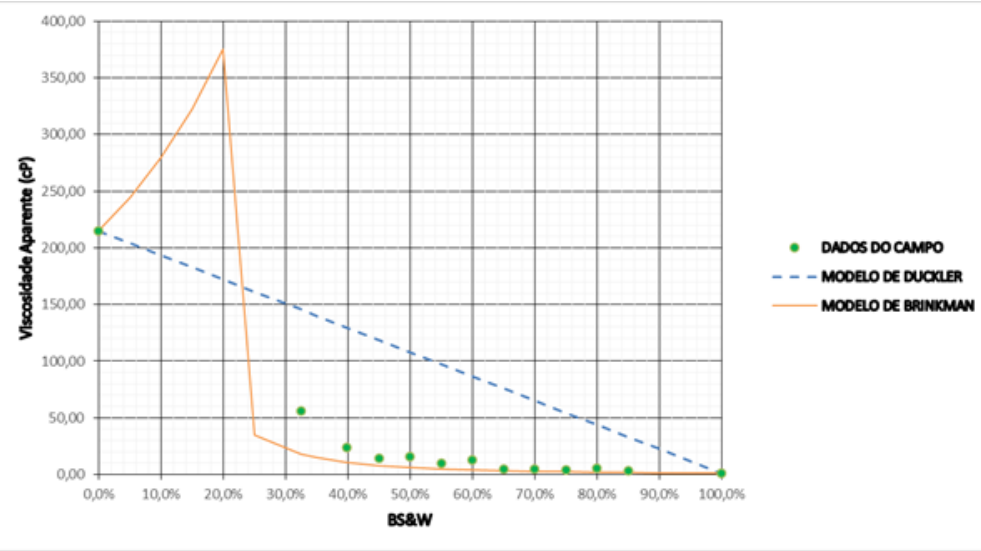

Figura 3 - Viscosidade aparente em função do BS\&W (dados de campo vs modelos). 
Como o modelo de Duckler usa uma média ponderada das viscosidades do óleo e da água era de se esperar que este não descrevesse bem a viscosidade da emulsão, principalmente na região mais próxima ao ponto de inversão de fase.

Já o modelo de Brinkman mostra uma boa aderência na região de alto BS\&W (acima de 70\%) mas há um desvio em relação a região próxima ao ponto de inversão de fase.

\section{CONCLUSÕES}

Foi realizado o estudo com os dados de escoamento em tubulações obtidos do campo, esses dados evidenciaram o comportamento de queda da viscosidade aparente do fluido com o aumento do BS\&W da emulsão. No entanto, ficou evidenciado também, a falta de dados de escoamento para a região de inversão de fase da emulsão.

Ainda assim, foi possível montar um mapa da viscosidade aparente por toda a região de escoamento levando em consideração algumas correlações obtidas da literatura. Enquanto a correlação de Brinkman apresentou resultados razoáveis, pelo menos em relação ao comportamento esperado, a correlação de Duckler mostrou-se inadequada para este tipo de sistema.

Uma investigação mais aprofundada pode ser levada em conta no caso da montagem de uma bancada de teste de escoamento em laboratório, dessa forma pode-se simular o escoamento real com a vantagem de trabalhar com uma faixa de BS\&W de operação mais adequada, além de isolar efeitos inerentes da operação que trazem o sistema a um desvio do regime permanente de escoamento.

\section{NOMENCLATURA}

D - diâmetro interno da tubulação em m.

e - rugosidade relativa (razão entre a rugosidade da tubulação e o diâmetro interno da mesma), adimensional.

$\mathrm{f}$ - fator de atrito, adimensional.

$\varepsilon_{\mathrm{W}}{ }^{\mathrm{I}}$ - fração volumétrica de água no ponto de inversão de fase, adimensional.

$\varepsilon_{\mathrm{W}}$ - fração volumétrica de água, adimensional.

$\varepsilon_{0}$ - fração volumétrica do óleo, adimensional.

$\mathrm{L}$ - comprimento da tubulação em $\mathrm{m}$.

$\rho$ - massa específica do fluido em $\mathrm{kg} / \mathrm{m}^{3}$.

$\rho_{\mathrm{w}}$ - massa específica da água em $\mathrm{kg} / \mathrm{m}^{3}$.

$\rho_{\mathrm{o}}$ - massa específica do óleo em $\mathrm{kg} / \mathrm{m}^{3}$.

$\Delta \mathrm{p}$ - perda de carga por fricção dada em $\mathrm{Pa}$.

Re - número de Reynolds, adimensional.

$\mathrm{v}$ - velocidade de escoamento em $\mathrm{m} / \mathrm{s}$.

$\mu$ - viscosidade dinâmica em Pa.s.

$\mu_{\mathrm{w}}$ - viscosidade dinâmica da água em Pa.s.

$\mu_{\mathrm{m}}$ - viscosidade dinâmica da mistura em Pa.s.

$\mu_{o}$ - viscosidade dinâmica do óleo em Pa.s. 
19 a 22 de outubro de 2014

Florianópolis/SC 


\section{REFERENCIAS}

ARIRACHAKARN, S., OGLESBY, K.D., BRILL, J.P., 1989. An Analysis of Oil/Water Flow Phenomena in Horizontal Pipes. SPE, p. 18836.

BASTIDAS, T. J. P. Geração de emulsões durante a produção de petróleo. 2007. 100 (Doutorado em Engenharia Mecânica). Engenharia Mecânica, Pontifícia Universidade Católica do Rio de Janeiro, Rio de Janeiro.

BRAUNER, N., ULLMANN, A., 2002. Modeling of phase inversion phenomenonin the twophase flows. Int. J. Multiph. Flow 28 (6), 1177-1204.

BRINKMAN, H., 1952. The viscosity of concentrated suspensions andsolutions. J. Chem. Phys. 20 (4), 571-584.

CHEN, J., 2001. Study on Oil-Water Two Phase Pipe Flow. University of Petroleum, Beijing, China.

DECARRE, S., FABRE, J., 1997. Phase inversion prediction study. Journal of L' Institute Francais du Prtrole, vol. 52, pp. 415-424.

DUKLER, A.E.,WICKS III, M., CLEVELAND, R.G., 1964. Frictional pressuredrop in two-phase flow. AIChE J. 10 (1), 44-51.

HAPANOWICZ, J. Slip between the phases in two-phase water-oil flow in a horizontal pipe. International Journal of Multiphase Flow, v. 34, p. 559-566, 2008.

KELEŞOĞLU, S.; PETTERSEN, B. H.; SJÖBLOM, J. Flow properties of water-in-North Sea heavy crude oil emulsions. Journal of Petroleum Science and Engineering, v. 100, n. 0, p. 14-23, 2012.

PLASENCIA, J.; PETTERSEN, B.; NYDAL, O. J. Pipe flow of water-in-crude oil emulsions: Effective viscosity, inversion point and droplet size distribution. Journal of Petroleum Science and Engineering, v. 101, n. 0, p. 35-43, 2013.

YEH, G., HAYNIE JR, F.H., MOSES, R.E., 1964. Phase-volumerelationship at the point of phase inversion in liquid dispersions.AIChE J. 10 (2), 260-265. 